\title{
Intermolecular Cooperativity and the Temperature Dependence of Segmental Relaxation in Semicrystalline Polymers
}

\author{
K. L. Ngai and C. M. Roland \\ Naval Research Laboratory, Washington, D.C. 20375-5342 \\ Received October 23, 1992; Revised Manuscript Received March 1, 1993
}

\begin{abstract}
The effect of the presence of a crystalline phase on the temperature dependence of segmental relaxation is examined. For both an aromatic and an aliphatic polyester, for polypropylene, and for poly(dimethylsiloxane) (PDMS), it is demonstrated that crystallinity has an insignificant effect on the temperature dependence of the segmental relaxation time when the temperature is normalized by the glass transition temperature, $T_{\mathrm{g}}$, of the polymer. This allows the correlation between time and temperature dependencies of segmental relaxation, whereby more intermolecularly cooperative relaxations have a stronger dependence on $T_{\mathrm{g}}$-normalized temperature, to continue to be used to assess the intermolecular coupling strength in semicrystalline polymers.
\end{abstract}

\section{Background}

Intuitively, the strength of the intermolecular coupling of the segmental relaxation of polymers should depend on the monomeric molecular structure. Steric constraints arising from bulky segments containing inflexible pendant moieties will enhance the dynamic coupling to nonbonded neighboring chain units. In the terminology of the coupling model of relaxation, ${ }^{1-3}$ this corresponds to a larger value of the coupling parameter, $n$, for segmental relaxation. By the same token, relaxation of segments which are flexible, compact, smooth, symmetric, etc. should be less intermolecularly constrained (smaller $n$ ). This consideration of only the relative effects of steric constraints on segmental relaxation is based on the assumption that the nature of the intermolecular potentials are similar. Obviously, modification of this potential (e.g., from London dispersion forces to strong polar interactions) will influence the degree of intermolecular cooperativity of the segmental dynamics.

In miscible blends of polymers the degree of intermolecular coupling associated with relaxation of a given segment will vary according to the composition of its local environment. ${ }^{4-9}$ While the effects of structure still exist, the inhomogeneous broadening due to concentration fluctuations may dominate the relaxation behavior. For example, if the surrounding segments are of lower $T_{\mathrm{g}}$ than the relaxing segment, the dynamic constraints imposed by these neighbors are mitigated. This allows segmental relaxation of the given segment to proceed with a lower degree of intermolecular cooperativity than would be the case in local environments richer in the higher $T_{\mathrm{g}}$ component.

Systematic studies concerning the effect of molecular structure on segmental relaxation of neat polymers are scarce. We have demonstrated the enhancement in intermolecular cooperativity of the segmental relaxation of polybutadiene as the vinyl content of the polymer is increased. ${ }^{10}$ The epoxidation of 1,4-polyisoprene to form random copolymers of 2-methyl-1,2-epoxy-1,4-butanediyl and 2-methyl-1-butenylene was similarly found to increase the strength of the intermolecular coupling of the segmental relaxation. ${ }^{11}$ Recently, the relatively unconstrained segmental relaxation of amorphous polytetrahydrofuran and polyisobutylene and its consequence on the viscoelastic properties have been described. ${ }^{12}$ These studies, each considering a class of polymers having intermolecular potentials of similar nature, are consistent with the correlation suggested above between structure (flexibility, compactness, smoothness, and symmetry of the backbone) and relaxation behavior.
The relationship between chemical structure and molecular motion is so central to polymer science that the idea that intermolecular cooperativity is governed by local structure must be more fully explored. The most direct method to assess the degree of intermolecular cooperativity is from the breadth of the segmental relaxation dispersion in the viscoelastic spectrum (measured, for example, by dielectric, mechanical, or dynamic light scattering spectroscopies). The out of phase component of the dynamic response is related to the relaxation function by ${ }^{13}$

$$
E^{\prime \prime}(\omega)=\omega \int_{0}^{\infty} E(t) \cos (\omega t) \mathrm{d} t
$$

where for neat, amorphous polymers, the latter usually has the Kohlrausch-Williams-Watts (KWW) form ${ }^{14-16}$

$$
E(t)=\left(E_{\mathrm{g}}-E_{\mathrm{r}}\right) \exp \left[-\left(\frac{t}{\tau^{*}(n)}\right)^{1-n}\right]+E_{\mathrm{r}}
$$

In this equation $\tau^{*}$ is an effective relaxation time, and $E_{\mathrm{g}}$ and $E_{\mathrm{r}}$ are the respective unrelaxed and relaxed moduli for segmental motion (a corresponding expression can be written in terms of the compliances). This form for the relaxation function can be derived by introducing intermolecular cooperativity into expressions for the relaxation of isolated chains. ${ }^{1-3}$ The coupling parameter $n$ increases with the breadth of the dispersion and, according to the coupling model, provides a measure of the strength of the intermolecular constraints on the relaxation.

The determination of $n$ by fitting eq 2 to experimental data has been carried out by numerous investigators, ${ }^{10,11,17}$ and none of these results is at odds with the interpretation of $n$ in terms of the strength of the intermolecular cooperativity. These data also are consistent with a prediction of the coupling model of a relation between time and temperature dependencies. ${ }^{18,19}$ Specifically, polymer chains whose segmental relaxation is characterized by stronger intermolecular coupling (larger $n$ ) are expected to exhibit a more marked dependence on temperature. This correlation of frequency and temperature dependencies has indeed been observed for a number of polymers. ${ }^{10-12,19}$ Of course, over the range of most experimental measurements, temperature dependencies are non-Arrhenius. This means that some normalization scheme must be invoked to allow comparisons among different polymers of the effect of temperature on the measured relaxation times. Angell ${ }^{20,21}$ proposed a normalization scheme employing $T_{\mathrm{g}}$-scaled Arrhenius plots of segmental relaxation times; that is, $\tau^{*}$ is plotted as a function of inverse temperature normalized by the glass 
transition temperature (anachronistically referred to as "fragility plots"; for polymers we have suggested "cooperativity plot" as a more appropriate terminology ${ }^{10}$ ). The validity of $T_{\mathrm{g}}$-scaled Arrhenius plots has recently been demonstrated by comparison of data from polymers differing only in molecular weight. ${ }^{22}$ For a number of polymers differing in chemical structure, it has been found that those with larger $n$ for segmental relaxation exhibit segmental relaxation times which change more with $T_{\mathrm{g}^{-}}$ normalized temperature, that is, steeper cooperativity curves. Moreover, wherever sufficient data exist, the correlations between molecular structure, intermolecular cooperativity, and temperature dependency appear to be valid. Of course, the interpretation of the normalized temperature dependence of $\tau^{*}$ solely in terms of intermolecular coupling strength is strictly valid only for polymers whose intramolecular constraints have similar temperature dependencies.

There are many polymers which crystallize and therefore render inapplicable the direct method of determining $n$ through fitting eq 2 . In these cases assessment of any correlation between structure and intermolecular coupling must rely on the influence of cooperativity on temperature dependencies (fragility). The main purpose of the present work is a demonstration that, while the shape of the segmental relaxation function, $E(t)$, is affected by crystallinity, the normalized temperature dependence of the segmental relaxation time is not. Having established this important point, the temperature dependence of segmental relaxation measured for semicrystalline polymers, along with that for amorphous polymers, can be used to develop the correlation between structure and intermolecular coupling strength.

\section{Crystallinity and Segmental Relaxation}

Previous studies of the effect of crystallization on segmental relaxation have shown that crystallization broadens the dispersion and shifts it to lower frequencies. ${ }^{23-26}$ This indicates that amorphous chain segments in proximity to the crystalline phase have their motions restricted by the latter. Their contribution to the measured response appears at lower frequencies, thus shifting the observed maximum in the dispersion to lower frequency. This inhomogeneously broadened dispersion no longer exhibits the KWW form (eqs 1 and 2); however, the temperature dependence may still be dominated by intermolecular cooperativity. Unfortunately, in highly crystalline polymers the $T_{\mathrm{g}}$ relaxation is usually too broad for the peak center (or a relaxation time) to be reliably determined. Demonstration of the effect of crystallization on the temperature dependence of segmental relaxation must rely on measurements from polymers of relatively low crystallinity.

Polyesters. The dielectric loss has been measured for PET crystallized to varying degrees. ${ }^{27}$ The presence of a crystalline phase markedly broadened the segmental dispersion, particularly toward lower frequency, resulting in a more symmetrical peak than the KWW form observed for amorphous PET. Neglecting the polymers crystallized under elevated pressure, the relaxation is displaced toward higher temperature in semicrystalline PET. ${ }^{27}$ Relaxation times, defined as the inverse frequency at the peak maximum, were obtained ${ }^{27}$ by fitting the experimental data to the empirical Havriliak-Negami equation. ${ }^{28}$ In Figure 1 the temperature dependence of these relaxation times is displayed in the cooperativity plot form for the amorphous polymer and for PET crystallized to various degrees at atmospheric pressure. The reference temper-

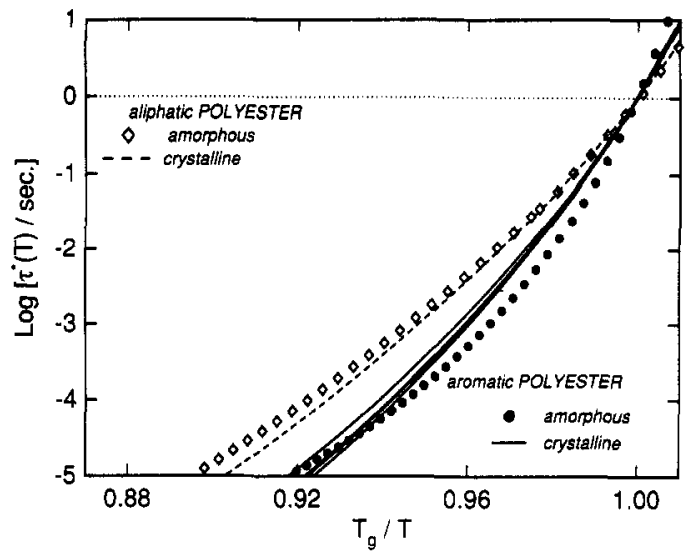

Figure 1. Cooperativity curve depictions of the temperature dependence on the relaxation times determined from dielectric spectroscopy (data taken from ref 27) on amorphous PET and PET crystallized to various degrees $(26 \%-50 \%)$, along with similar data for two aliphatic polyesters of similar chemical structure, but differing in crystallinity (data from ref 29 ).

ature $T_{\mathrm{g}}$, defined as the temperature at which $\tau=1 \mathrm{~s}$, increases from $353 \mathrm{~K}$ for amorphous PET to as much as $367 \mathrm{~K}$ in a PET which is $29 \%$ crystalline. When the temperatures are normalized by $T_{\mathrm{g}}$, the temperature dependence of the relaxation times is seen to vary very insubstantially with crystallization. This indicates that while the constraints on segmental motion increase the relaxation time of chain units in proximity to crystallites, the degree of intermolecular cooperativity and, hence, the temperature dependence of the relaxation are unaltered. Thus, for inhomogeneously broadened dispersions the intermolecular coupling strength can be assessed from the temperature dependence of the relaxation.

Also shown in Figure 1 are data from an amorphous polyester obtained by the condensation of dimethyl adipate with 2,5-hexanediol, along with a semicrystalline random copolymer obtained by condensation of the dimethyl adipate with a $60 / 40$ mole ratio of 1,6- and 2,5-hexanediol. ${ }^{29}$ The similarity of the chemical structure of these aliphatic polyesters implies a comparable degree of intermolecular cooperativity for their segmental relaxations, although this cannot be assessed directly from the segmental dispersion due to inhomogeneous broadening in the semicrystalline polymer. Since the normalized temperature dependence is essentially unaffected by crystallinity, the comparable degree of intermolecular cooperativity of their segmental relaxations is confirmed by the near equivalence of their cooperativity plots.

Polypropylene. Isotactic polypropylene crystallizes, unlike the atactic isomer. In Figure 2 normalized Arrhenius plots of the retardation times from dynamic light scattering are displayed for amorphous, atactic polypropylene, ${ }^{30}$ along with the relaxation times extracted from dynamic mechanical measurements on the semicrystalline isotactic polypropylene. ${ }^{31}$ Despite the very different shape of their respective relaxation functions (the semicrystalline polymer having a broader dispersion), the normalized temperature dependencies are equivalent. This result is in accord with the equivalent degree of intermolecular coupling expected for segmental relaxation within the respective amorphous phases.

Poly(dimethylsiloxane). In Figure 3 are dielectric spectroscopy results of segmental relaxation times measured at different temperatures on amorphous and semicrystalline PDMS. ${ }^{32}$ Although the Arrhenius plots deviate from linearity, activation energies for the glass transition were reported, and these differed for the amorphous and 


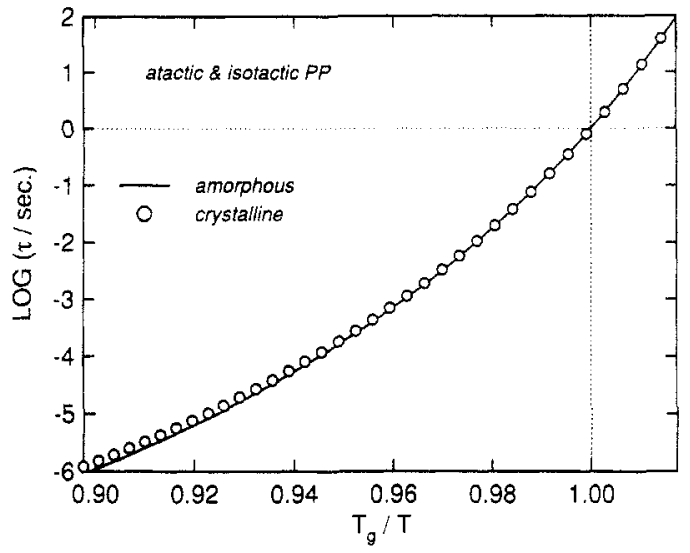

Figure 2. Normalized Arrhenius plots of the segmental relaxation times measured for amorphous, atactic polypropylene, ${ }^{30}$ along with the relaxation times extracted from dynamic mechanical measurements on semicrystalline isotactic polypropylene. ${ }^{31}$

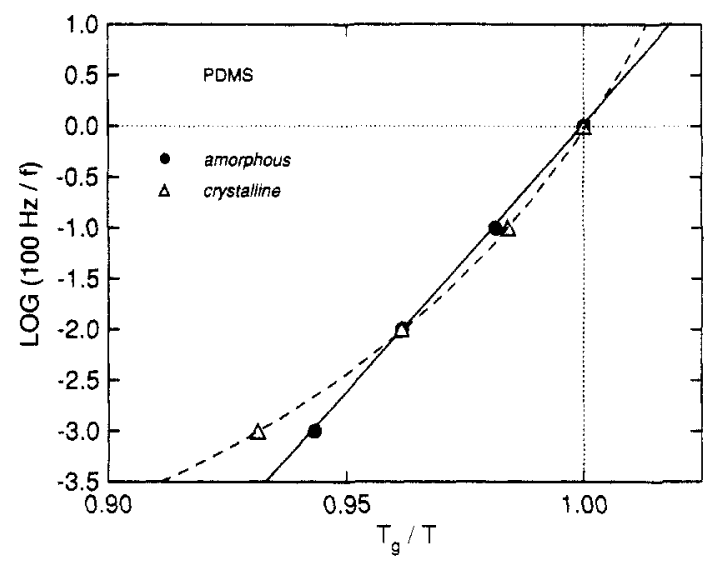

Figure 3. Frequency (in Hertz) of the maximum in the dielectric loss measured at different temperatures on amorphous and semicrystalline poly (dimethylsiloxanes) (data taken from ref 32 ).

semicrystalline forms. However, when expressed in the $T_{\mathrm{g}}$-scaled Arrhenius form, the temperature dependencies are seen to be quite comparable.

\section{Summary}

A plethora of results has been published demonstrating the value of the coupling scheme of relaxation, and interpretations and predictions of the model are unfailingly in accord with experimental results. The model's principal result is that intermolecular cooperativity in dense phase gives rise to the factor $\left(\omega_{c} t\right)^{-n}$ retarding the relaxation rate of macroscopic variables, where the coupling parameter, $n$, scales with the intermolecular coupling strength. This result leads to a number of predictions shown to be in agreement with experimental data. ${ }^{10-12,19,33-35}$ These studies show that the coupling parameter $n$ governs various properties involving segmental relaxation. The derivations of the coupling model make clear that the magnitude of $n$ is determined by the degree of intermolecular coupling. For a given pair of polymers, physical arguments concerning their respective repeat unit structures can be used to deduce which should have stronger intermolecular coupling. In turn, from the dependence on $n$ of various segmental relaxation properties, predictions can be made regarding the trend (or relative magnitude) for any such property in going from one polymer to the other.

It has been shown herein that, for semicrystalline polymers, the shape of the segmental dispersion associated with intermolecular coupling is masked by inhomogeneous broadening. Consequently, the relaxation functions are not easily interpretable and coupling parameters cannot be deduced directly. Notwithstanding, crystallinity exerts a negligible influence on the $T_{\mathrm{g}}$-scaled temperature dependence of the segmental relaxation time, particularly as determined from the shift of the loss peak, allowing assessments to be made of the extent of intermolecular cooperativity. In the future we will exploit this approach to examine in various semicrystalline polymers, as well as amorphous polymers, the variation of intermolecular coupling with molecular structure. By extending the analysis to segmental relaxation data from a wide range of polymers, our intention is to develop physical intuitions concerning the manner in which chemical structure governs segmental dynamics.

Acknowledgment. This work was supported by the Office of Naval Research; K.L.N., in part by Contract N0001492WX24029.

\section{References and Notes}

(1) Ngai, K. L.; Rendell, R. W.; Rajagopal, A. K.; Teitler, S. Ann. N.Y. Acad. Sci. 1986, 484, 150.

(2) Ngai, K. L.; Rendell, R. W. J. Non-Cryst. Solids 1991; 131-133, 942.

(3) Ngai, K. L.; Peng, S. L.; Tsang, K. Y. Physica A 1992, 191, 523.

(4) Trask, C. A.; Roland, C. M. Macromolecules 1989, 22, 256.

(5) Miller, J. B.; McGrath, K. J.; Roland, C. M.; Trask, C. A.; Garroway, A. N. Macromolecules 1990, 23, 4543.

(6) Roland, C. M.; Ngai, K. L. Macromolecules 1991, 24, 2261.

(7) Roland, C. M.; Ngai, K. L. Macromolecules 1992, 25, 363.

(8) Roland, C. M.; Ngai, K. L. J. Rheol. 1992, 36, 1691.

(9) Roland, C. M.; Ngai, K. L.; O'Reilly, J. M.; Sedita, J. S. Macromolecules 1992, 25, 3906.

(10) Roland, C. M.; Ngai, K. L. Macromolecules 1991, 24, 5315.

(11) Roland, C. M. Macromolecules 1992, 25, 7031.

(12) Santangelo, P. G.; Ngai, K. L.; Roland, C. M. Macromolecules, in press.

(13) Ferry, J. D. Viscoelastic Properties of Polymers; Wiley: New York, 1980

(14) Kohlrausch, R. Pogg. Ann. Phys. 1847, 12, 393.

(15) Williams, G.; Watts, D. C. Trans. Faraday Soc. 1970, 66, 80.

(16) Ngai, K. L. In Non-Debye Relaxation in Condensed Matter; Ramakrishnan, T. V., Lakshmi, M. R., Eds.; World Scientific: Singapore, 1988; p 94.

(17) See papers in: J. Non-Cryst. Solids 1991, 131-133.

(18) Ngai, K. L.; Rendell, R. W.; Plazek, D. J. J. Chem. Phys. 1991, $94,3018$.

(19) Plazek, D. J.; Ngai, K. L. Macromolecules 1991, 24, 1222.

(20) Angell, C. A. In Relaxations in Complex Systems; Ngai, K. L., Wright, G. B., Eds.; Government Printing Office: Washington, DC, $1985 ; \mathrm{p} 3$.

(21) Angell, C. A. J. Non-Cryst. Solids 1991, 131-133, 13.

(22) Roland, C. M.; Ngai, K. L. Macromolecules 1992, 25, 5765.

(23) McCrum, N. G.; Read, B. E.; Williams, G. Anelastic and Dielectric Effects in Polymer Solids; Wiley: London, 1967.

(24) Wetton, R. E.; Allen, G. Polymer 1966, 7, 331.

(25) Sawada, K.; Ishida, Y. Rep. Prog. Poly. Phys. Jpn. 1974, 17, 437.

(26) Boyd, R. H. Polymer 1985, 26, 323.

(27) Coburn, J. C.; Boyd, R. H. Macromolecules 1986, 19, 2238.

(28) Havriliak, S.; Negami, S. J. Poly. Sci. C 1966, 14, 99.

(29) Boyd, R. H.; Aylwin, P. A. Polymer 1984, 25, 331.

(30) Fytas, G.; Ngai, K. L. Macromolecules 1988, 21, 804.

(31) Read, B. E. Polymer 1989, 30, 1439.

(32) Adachi, H.; Adachi, K.; Ishida, Y.; Kotaka, T. J. Polym. Sci., Polym. Phys. Ed. 1979, 17, 851.

(33) Ngai, K. L.; Plazek, D. J. J. Polym. Sci., Polym. Phys. Ed. 1986, 24,619 .

(34) Plazek, D. J.; Zhang, X. D.; Ngai, K. L. Macromolecules, in press.

(35) Ngai, K. L.; Colmenero, J.; Alegria, A.; Arbe, A. Macromolecules, in press. 